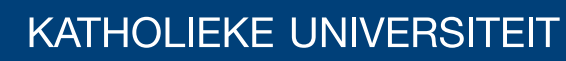 \\ LEUVEN
}

\section{Faculty of Business and Economics}

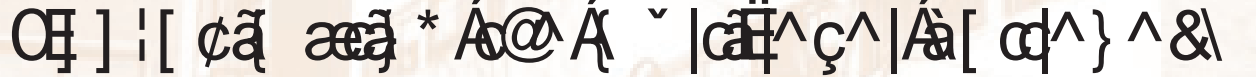

\section{DWJ QP HQLSLREOP}

7 UMNDP [' RWDI\$ QDWDVDI. RXYHODDQG) UNI6 SLHMP D

DEPARTMENT OF DECISION SCIENCES AND INFORMATION MANAGEMENT (KBI) 


\title{
Approximating the Multi-Level Bottleneck Assignment Problem*
}

\author{
Trivikram Dokka ${ }^{1}$, Anastasia Kouvela ${ }^{2}$, and Frits C.R. Spieksma ${ }^{1}$ \\ 1 ORSTAT, K.U.Leuven, Naamsestraat 69, B-3000 Leuven, Belgium. \\ trivikram.dokka;frits.spieksma@econ.kuleuven.be. \\ 2 Management Science Group, London School of Economics, UK.
}

\begin{abstract}
We consider the multi-level bottleneck assignment problem (MBA). This problem is described in the recent book "Assignment Problems" by Burkard et al. (2009) on pages 188 - 189. One of the applications described there concerns bus driver scheduling. We view the problem as a special case of a bottleneck $m$-dimensional multi-index assignment problem. We give approximation algorithms and inapproximability results, depending upon the completeness of the underlying graph.

Keywords: bottleneck problem; multidimensional assignment; approximation; computational complexity; efficient algorithm.
\end{abstract}

\section{Introduction}

We consider an $m$-dimensional bottleneck assignment problem. Let $V_{1}, V_{2}, \ldots, V_{m}$ be pairwise disjoint sets, each with cardinality $n$, and let $V=\cup_{i=1}^{m} V_{i}$. There is a given weight $w(v) \in \mathbb{N}$ for each $v \in V$. The set $V$ is the node-set of an $m$-partite graph that has a given set of $\operatorname{arcs} E$ of the following form: $E=\{(u, v) \mid u \in$ $\left.V_{i}, v \in V_{i+1}\right\}$. Thus, an arc in $E$ connects a node from $V_{i}$ with a node from $V_{i+1}(1 \leq i \leq m-1)$, and there are no other arcs. A feasible $m$-tuple (to which we will refer as a duty) is a set of nodes $D=\left\{v_{1}, v_{2}, \ldots, v_{m}\right\}$ such that $v_{i} \in V_{i}$ for $1 \leq i \leq m$, and such that $\left(v_{i}, v_{i+1}\right) \in E$. The cost of a duty $D$ equals $c(D)=\sum_{v \in D} w(v)$. The problem is to find a partition of $V$ into $n$ duties $D_{1}, D_{2}, \ldots, D_{n}$ such that $\max _{j} c\left(D_{j}\right)$ is minimum. We will refer to this partition of $V$ into $\left\{D_{1}, D_{2}, \ldots, D_{n}\right\}$ as a solution $S$, and the cost of a solution $S$ equals $c(S)=\max _{j}\left\{c\left(D_{j}\right) \mid S=\left\{D_{1}, D_{2}, \ldots, D_{n}\right\}\right\}$.

This problem is known as the multi-level bottleneck assignment problem (MBA); obviously, for $m=2$ a (special case of the) bottleneck assignment problem arises. It was introduced by Carraresi and Gallo [6], motivated by an application in bus driver scheduling. In the context of this application, a set $V_{i}$ corresponds to the shifts that need to be carried on day $i(1 \leq i \leq m)$; further, an edge $\left(v_{i}, v_{i+1}\right) \in E$ indicates that it is possible to perform shift $v_{i+1}$ directly

\footnotetext{
* This research was supported by OT Grant OT/07/015.
} 
after shift $v_{i}(1 \leq i \leq m-1)$. Then, a duty $D_{j}$ is a set of shifts, one from each day, to be carried out by a driver. The cost of a duty is nothing else but the load of a driver, and the goal is to minimize the maximum load. Notice that [6] phrase the problem using a weight $w(v)$ on each arc leaving $v$, when $v \in \cup_{i=1}^{m-2} V_{i}$, and an arc with weight $w(u)+w(v)$ for each arc $(u, v) \in V_{m-1} \times V_{m}$. They show that the problem is NP-hard when $m$ is part of the input by a reduction from Even-Odd Partition, and they leave as an open problem the complexity for a fixed $m$. This problem MBA is also described in the recent book of Burkard et al. [4], in which it is stated that the complexity of this problem is unresolved for each fixed $m \geq 3$ (pages 188-189); we will settle this question in Section 2.

\section{Related Work}

As described above, the problem seems to be first introduced in [6], who, in addition to proving NP-completeness for arbitrary $m$, also describe a heuristic with computational experiments. Another heuristic, with better computational results, is given in Bianco et al. [2]. A generalization of this problem has been considered computationally in Cappanera and Gallo [5], and a further extension is recently considered in Aringhieri and Cordone [1].

We observe here that MBA can also be seen as a generalization of the multiprocessor scheduling problem, where one allows for incompatibilities between jobs. Indeed, when viewing each node $v \in V$ as a job with $w(v)$ its processing time, and each duty as a machine, then a multi-processor scheduling problem arises with the constraint that nodes (jobs) from the same set $V_{i}$ should not be assigned to the same duty (machine). Such problems have been studied in Bodlaender et al. [3]; however, their results do not apply to MBA. Approximation schemes have been proposed for related problems. Jansen [9] describes a APTAS for the bin packing problem with conflicts. Notice however, that in our problem, the number of bins is fixed; instead, the problem is to minimize the maximum weight.

An important special case of MBA is the case where $E$ is complete, i.e., in terms of the application, the case where each shift from day $V_{i}$ can be directly followed by each shift from day $V_{i+1}$. We will call this special case complete$M B A$. In fact, this special case was studied by Hsu [8] and by Coffman and Yannakakis [10] from an approximation point of view. For complete-MBA, Hsu [8] gave an $\left(2-\frac{1}{n}\right)$-approximation algorithm that runs in $O(m n \log n)$, while Coffman and Yannakakis [10] gave an $O\left(n^{2} m\right)\left(\frac{3}{2}-\frac{1}{2 n}\right)$-approximation algorithm. For the case where $m=3$, Hsu gave a $\frac{3}{2}$-approximation algorithm that runs in $O(n \log n)$, and a $\frac{4}{3}$-approximation algorithm that runs in $O\left(n^{3} \log n\right)$. We will refer to our problem MBA with $m=3$ as MBA3. Three-dimensional bottleneck assignment problems with more general cost structures than MBA3 have been studied by Klinz and Woeginger [11], and more recently by Goossens et al. [7].

As far as we are aware, all known approximation results deal with the case where every pair of nodes of different sets can be joined in a duty, i.e., the case of complete-MBA. In this paper we deal with a more general setting, namely 


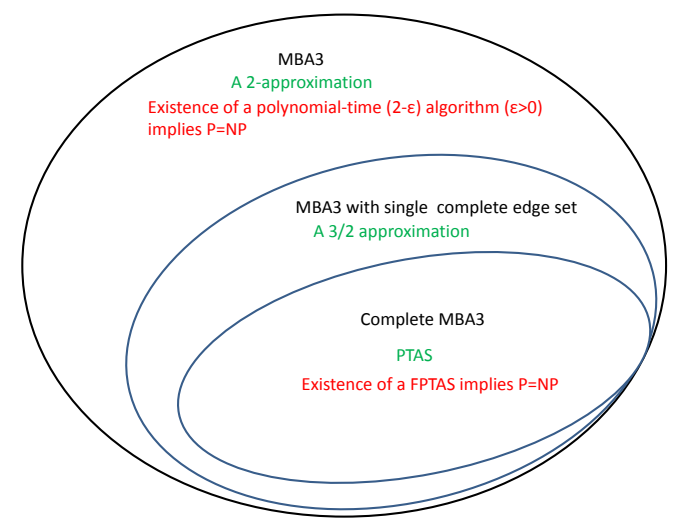

Fig. 1. Overview of results for $M B A 3$

the case where the edge set between $V_{i}$ and $V_{i+1}$ can be arbitrary (and not necessarily complete) $(1 \leq i \leq m-1)$. In fact, we see as our main contribution exploring the approximability of MBA depending upon whether or not the edge sets between $V_{i}$ and $V_{i+1}$ are assumed to be complete. When we restrict ourselves to $m=3$, notice that there is an edge set between $V_{1}$ and $V_{2}$, denoted by $E_{1}$, and an edge set between $V_{2}$ and $V_{3}$, denoted by $E_{2}$. Thus, when $m=3$, we can distinguish three cases:

- both $E_{1}$ and $E_{2}$ are complete (complete-MBA3),

- $E_{1}$ or $E_{2}$ (or both) is complete (MBA3 with a single complete edge set), and

- both $E_{1}$ and $E_{2}$ are arbitrary (MBA3).

Observe that an instance of MBA3 is such that any node from $V_{1}$ can be joined in a duty with any node from $V_{3}$. In case there would be restrictions on that as well, the resulting decision version of MBA3 would be equivalent to 3DM.

\section{Our Results}

- There is a simple 2-approximation algorithm for MBA3 (see Section 3.1), while the existence of a $(2-\epsilon)$-polynomial time approximation algorithm for MBA3 $(\epsilon>0)$ implies $\mathrm{P}=\mathrm{NP}$ (see Section 3.2),

- There is $\frac{3}{2}$-approximation algorithm for MBA3 with a single complete edge set (see Section 4),

- There is a PTAS for complete-MBA for each $m \geq 3$ (see Section 5), while complete-MBA3 is strongly NP-hard (see Section 2),

- Online MBA can be seen as a generalisation of online parallel machine scheduling (see Section 6).

See Figure 1 for an overview of our results for MBA3. Notice that for completeMBA3, and for MBA3, the proposed algorithms are best-possible. 


\section{The complexity of MBA}

As already observed by [6], deciding feasibility of an instance of MBA is not a difficult problem: by verifying whether the arc set $E$ contains a bipartite matching between each pair of sets $\left(V_{i}, V_{i+1}\right), 1 \leq i \leq m-1$, it follows whether there exists a feasible solution. Finding a best solution, however, is a more difficult problem, even for $m=3$, and even when the edge sets are complete. We will now informally argue that complete-MBA3 is at least as hard as Numerical 3-Dimensional Matching.

We first describe Numerical 3-Dimensional Matching (N3DM), which is known to be NP-hard. Numerical 3-Dimensional Matching has as input 3 sets of positive integers $x_{1}, \ldots, x_{n}$ (the set $X$ ), $y_{1}, \ldots, y_{n}$ (the set $Y$ ), and $z_{1}, \ldots, z_{n}$ (the set $Z)$, and a bound $B$ such that $\sum_{i=1}^{n}\left(x_{i}+y_{i}+z_{i}\right)=n B$. The question is whether there exist $n$ disjoint triples, each containing one element from each of the three sets, such that for each triple $x_{i}+y_{j}+z_{k}=B$.

Given an instance of N3DM we now build, in a straightforward manner, an instance of our problem. Let the number of shifts equal $n$, and the number of days equal 3, i.e., $m:=3$. We assume that all arcs are present, i.e., $E:=$ $\left\{(u, v) \mid(u, v) \in\left(V_{1} \times V_{2}\right) \cup\left(V_{2} \times V_{3}\right)\right\}$. Further, the weight of a node $v_{i} \in V_{1}$ (or, in terms of [6], the weight of an arc leaving a node in $V_{1}$ ), equals $x_{i}$, thus $w\left(v_{i}\right)=x_{i}$ for each $v_{i} \in V_{1}$. Similarly, we have $w\left(v_{i}\right):=y_{i}$ for each $v_{i} \in V_{2}$, and $w\left(v_{i}\right):=z_{i}$ for each $v_{i} \in V_{3}$ (or, when phrased in terms of [6], the weight of an arc leaving node $v_{i} \in V_{2}$, going to node $v_{i^{\prime}} \in V_{3}$ equals $y_{i}+z_{i^{\prime}}\left(1 \leq i, i^{\prime} \leq n\right)$ ). The question is: does there exist a solution consisting of $n$ duties such that the maximum load of a duty is no more than $B$ ? This completes the description of an instance of MBA. It is now easy to verify that a yes-instance of N3DM corresponds to a solution of our problem with $\operatorname{cost} B$, and vice versa. We record the above discussion by stating the following fact (which settles the question in $[4])$.

Fact 1 complete-MBA3 is NP-hard.

\section{The approximability of MBA3}

In this section we focus on MBA3. We present a simple 2-approximation algorithm called Sequential Bottleneck (SB) in Section 3.1, and we show in Section 3.2 that this approximation factor is, in fact, best possible. 


\subsection{The Sequential Bottleneck Heuristic (SB)}

SB runs in two stages: In the first stage, SB computes a bottleneck matching between $V_{1}$ and $V_{2}$. More precisely, the following integer program is solved.

$$
\begin{aligned}
& \min \max _{u \in V_{1}, v \in V_{2}}(w(u)+w(v)) x_{u, v} \\
& \text { s.t. } \quad \sum_{v:\{u, v\} \in E} x_{u, v}=1 \quad \text { for each } u \in V_{1} \\
& \sum_{u:\{u, v\} \in E} x_{u, v}=1 \quad \text { for each } v \in V_{2} \\
& x_{u, v} \in\{0,1\} \quad \text { for each }\{u, v\} \in E .
\end{aligned}
$$

Let $M$ denote the resulting matching, i.e., $M=\left\{(u, v) \mid x_{u, v}^{*}=1\right\}$, and let $w(M)$ denote the weight of this matching. In the second stage, SB computes a bottleneck assignment between the pairs in $M$ and the elements in $V_{3}$, with as a cost the cost of the resulting triple. Thus, we solve an integer program that is similar to the one above, where we replace $u \in V_{1}$ by $(u, v) \in M$, and where we replace $v \in V_{2}$ by $z \in V_{3}$. When heuristic SB is run on some instance $I$, we denote the cost of the resulting solution by $\mathrm{SB}(I)$.

Theorem 2. Heuristic SB is a polynomial-time, 2-approximation algorithm for MBA3. Moreover, there exist instances for which this bound is tight.

Proof. Obviously, SB is a polynomial-time algorithm, since it amounts to solving two bottleneck assignment problems (we refer to [4] for achievable time-bounds; when $E_{1}$ and $E_{2}$ are complete, simply sorting the weights suffices to solve the bottleneck assignment problems).

Consider now the solution found by SB; let its cost be determined by triple $(u, v, z) \in V_{1} \times V_{2} \times V_{3}$. Then:

$$
S B(I)=w(u)+w(v)+w(z) \leq w(M)+\max _{z \in V_{3}} w(z) .
$$

Further, it is easily seen that $\mathrm{OPT} \geq w(M)$, and that $\mathrm{OPT} \geq \max _{z \in V_{3}} w(z)$, where $O P T$ refers to the cost of an optimal solution. The result follows.

Finally, consider the following example, where we - with a slight abuse of notation - identify elements of a set with their weights: $V_{1}=\{n-1, \ldots, 1,0\}$, $V_{2}=\{n-1, \ldots, 1,0\}$ and $V_{3}=\{n, 0, \ldots, 0\}$. The edge sets $E 1$ and $E_{2}$ are complete. The value of an optimal solution is $n$, while SB gives a solution with value $2 n-1$.

Notice that SB detects whether an instance has a feasible solution. Also, notice that in order to obtain a ratio of 2 , any assignment in the second stage suffices. And although even more elaborate algorithms than SB can certainly be conceived, no polynomial time algorithm can improve upon the factor of 2 (unless $\mathrm{P}=\mathrm{NP}$ ), as we show next. 


\subsection{An inapproximability result}

We show that MBA3 cannot be approximated within a factor of 2 unless $\mathrm{P}=\mathrm{NP}$. To do so, we use a traditional technique: we will show that a YES-instance of 3 -dimensional matching (3DM) corresponds to an instance of MBA3 with cost 1, whereas a NO-instance corresponds to an instance of our problem with cost 2. Then, a polynomial time approximation algorithm with a worst case ratio strictly less than 2 would be able to distinguish the YES-instances of 3DM from the NO-instances, and this would imply $\mathrm{P}=\mathrm{NP}$.

Let us first recall 3-dimensional matching.

Instance: Three sets $X=\left\{x_{1}, \ldots, x_{q}\right\}, Y=\left\{y_{1}, \ldots, y_{q}\right\}$, and $Z=\left\{z_{1}, \ldots, z_{q}\right\}$, and a subset $T \subseteq X \times Y \times Z$.

Question: does there exist a subset $T^{\prime}$ of $T$ such that each element of $X \cup Y \cup Z$ is in exactly one triple of $T^{\prime}$ ?

Let the number of triples be denoted by $|T|=p$. Further, let the number of triples in which element $y_{j}$ occurs, be denoted by \#occ $\left(y_{j}\right), j=1, \ldots, q$.

Starting from arbitrary instance of 3DM, we now build a corresponding instance of MBA3 by specifying $V_{i}(i=1,2,3), E$, and the weights $w$ as follows:

- for each triple in $T$, there is a node in $V_{2}$. We refer to these nodes as triple nodes.

- for each $x_{i} \in X$, there is a node in $V_{1}(i=1, \ldots, q)$. In addition, for each $y_{j} \in Y$, there are \#occ $\left(y_{j}\right)-1$ nodes in $V_{1}(j=1, \ldots, q)$; for such a node in $V_{1}$ we say that this node corresponds to element $y_{j}$. These latter nodes will be referred to as the dummy nodes of $V_{1}$.

- for each $z_{k} \in Z$, there is a node in $V_{3}(k=1, \ldots, q)$. Further, we have $p-q$ additional nodes in $V_{3}$. These latter nodes will be referred to as the dummy nodes of $V_{3}$.

Notice that this construction ensures that $\left|V_{2}\right|=\left|V_{1}\right|=\left|V_{3}\right|=p$.

Let the nodes of $V_{1}, V_{2}$, and $V_{3}$ be denoted by $\left\{x_{1}^{\prime}, \ldots, x_{p}^{\prime}\right\},\left\{t_{1}^{\prime}, \ldots, t_{p}^{\prime}\right\}$, and $\left\{z_{1}^{\prime}, \ldots, z_{p}^{\prime}\right\}$ respectively. Thus, $\left\{x_{1}^{\prime}, \ldots, x_{q}^{\prime}\right\}$ are the non-dummy nodes of $V_{1}$, and $\left\{x_{q+1}^{\prime}, \ldots, x_{p}^{\prime}\right\}$ are the dummy nodes of $V_{1}$; notice that each dummy node of $V_{1}$ corresponds to some element $y_{j} \in Y$. Further, triple nodes $\left\{t_{1}^{\prime}, \ldots, t_{p}^{\prime}\right\}$ simply correspond to the triples in $T$, while $\left\{z_{1}^{\prime}, \ldots, z_{q}^{\prime}\right\}$ are the non-dummy nodes of $V_{3}$, and $\left\{z_{q+1}^{\prime}, \ldots, z_{p}^{\prime}\right\}$ are the dummy nodes of $V_{3}$. The edge set $E$ is defined as follows:

- There is an edge $\left(x_{i}^{\prime}, t_{j}^{\prime}\right)$ if $x_{i}$ is in the $j$-th triple in $T$, for $i=1, \ldots, q$ and $j=1, \ldots, p$.

- There is an edge $\left(t_{j}^{\prime}, z_{k}^{\prime}\right)$ if $z_{k}$ is in the $j$-th triple in $T$, for $k=1, \ldots, q$ and $j=1, \ldots, p$.

- There is an edge $\left(t_{j}^{\prime}, z_{k}^{\prime}\right)$, for $j=1, \ldots, p$ and $k=q+1, \ldots, p$.

- There is an edge $\left(x_{i}^{\prime}, t_{k}^{\prime}\right)$ if element $y_{j} \in Y$, to which dummy node $x_{i}^{\prime}$ corresponds, is contained in the $k$-th triple of $T$. 
To complete the description of our instance of MBA3, we assign the weights to the nodes in $V_{1}, V_{2}$, and $V_{3}$ as follows. All weights are zero, except the weights of the dummy nodes in $V_{1}$, and the weights of the non-dummy nodes in $V_{3}$ : these weights equal 1 .

Lemma 1. If the instance of $3 D M$ is a YES-instance, the corresponding instance of MBA3 has cost 1 . If the instance of $3 D M$ is a NO-instance, the corresponding instance of MBA3 has cost 2.

Proof. Suppose that the instance of 3DM is a YES-instance. Then we construct a solution to MBA3 as follows. First, we copy each of the $q$ triples in $T^{\prime}$ to duties in our solution of MBA3 by selecting the corresponding triple node $t_{j}^{\prime}$ in $V_{2}$, together with the associated nondummy node $x_{i}^{\prime}$ from $V_{1}$ and nondummy node $z_{k}^{\prime}$ from $V_{3}$ (notice that the corresponding edges are in $E$ ). The resulting $q$ duties contain all nondummy nodes in $V_{1}$ as well as all nondummy nodes in $V_{3}$. Further, we build duties containing the dummy nodes in $V_{1}$ by assigning each such node to the triple node in $V_{2}$ that contains element $y_{j}$ corresponding to the dummy node in $V_{1}$. This is always possible, since the instance of $3 \mathrm{DM}$ is a YES-instance, and hence, for each $y_{j} \in Y$, exactly \#occ $\left(y_{j}\right)-1$ nodes in $V_{2}$ remain. Since the edge set $E$ contains any edge between a dummy node in $V_{3}$ and a node in $V_{2}$, we can extend these pairs to duties by assigning the dummy nodes in $V_{3}$ to these pairs. Observe that each resulting duty has cost 1.

Conversely, consider a solution to this instance of MBA3 with cost 1 . This means that no non-dummy node from $V_{3}$ is in a duty with a dummy node from $V_{1}$. Consider the $p-q$ duties that contain the dummy nodes of $V_{1}$ : these duties will also contain all dummy nodes of $V_{3}$ (otherwise the cost of this solution would exceed 1). The remaining $q$ duties of our solution must be such that each of them contains a triple node from $V_{2}$, a nondummy node from $V_{1}$, and a nondummy node from $V_{3}$. These duties correspond to $q$ triples that define a solution to $3 \mathrm{DM}$.

Notice that the instances we have constructed are special in the sense that all weights are in $\{0,1\}$. In addition, the arguments go through when the degree of each node is bounded by some constant. Based on Lemma 1, and on the preceding discussion we can now state:

Theorem 3. There is no polynomial time algorithm for $M B A 3$ that achieves an approximation guarantee of $2-\epsilon$, for any $\epsilon>0$, unless $P=N P$.

Observe that the instances we constructed are such that neither $E_{1}$ nor $E_{2}$ are complete. This is a necessary property of these instances, as witnessed by the result in the next section.

\section{A $\frac{3}{2}$-approximation algorithm for MBA3 with a single complete edge set}

In this section we present a $\frac{3}{2}$-approximation algorithm for instances of MBA3 where the edge set $E_{2}$ (i.e., the edge set between $V_{2}$ and $V_{3}$ ) is arbitrary and the 
edge set $E_{1}$ (i.e., the edge set between $V_{1}$ and $V_{2}$ ) is complete. Notice that, for complete-MBA3, both [8] and [10] present $\frac{3}{2}$-approximation algorithms. These heuristics, however, do not seem to be generalizable to MBA3 with a single complete edge set while preserving the approximation factor.

We will call our algorithm heuristic AB (first Assign, then Bottleneck). In the description of $\mathrm{AB}$, we first assume that the value of the optimal solution, called $O P T$, is known. Without loss of generality, we further assume that the nodes in $V_{1}, V_{2}$, and $V_{3}$ are ordered in non-increasing order of their weights, with $V_{1}=\left\{u_{1}, u_{2}, \ldots, u_{n}\right\}$ and $V_{2}=\left\{v_{1}, v_{2}, \ldots, v_{n}\right\}$. We say that a node $u \in V$ is heavy if $w(u)>\frac{O P T}{2}$, and we call $u$ non-heavy otherwise. Let $k_{i}$ be the number of heavy nodes in $V_{i}, i=1,2,3$; notice that $k_{1}+k_{2}+k_{3} \leq n$.

\subsection{The description of heuristic AB}

Heuristic $\mathrm{AB}$ has two stages. In the first stage, we solve an instance of the maximum weight perfect matching problem on a bipartite graph $G^{\prime}=\left(V_{2}, V_{3}, E_{2}^{\prime}\right)$. The edge set $E_{2}^{\prime}$ is defined as follows: there is an edge $(v, z) \in E_{2}^{\prime}$ (with $(v, z) \in$ $\left.V_{2} \times V_{3}\right)$, if (i) $(v, z) \in E_{2}$, and (ii) $w(v)+w(z) \leq O P T$. Further, we define the weight $w^{\prime}$ of an edge $(v, z) \in E_{2}^{\prime}$ as follows:

$$
\begin{aligned}
w^{\prime}(v, z)=1 & \text { if } w(v)+w(z) \leq O P T / 2 \\
=0 & \text { otherwise }
\end{aligned}
$$

In the second stage, we compute a bottleneck matching (see Section 3.1) between the nodes from $V_{1}$ and the $n$ pairs found in Stage 1; this gives us the solution of $\mathrm{AB}$.

\subsection{The analysis of heuristic AB}

Theorem 4. Heuristic $A B$ is a polynomial-time $\frac{3}{2}$-approximation algorithm for $M B A 3$ with a single complete edge set. Moreover, there exist instances for which this bound is tight.

Proof. To begin with: AB is a polynomial-time algorithm. The first stage amounts to solving a maximum weight bipartite perfect matching problem, which can be done in polynomial time.

Let us next argue that heuristic $\mathrm{AB}$ finds a feasible solution whenever one exists. Indeed, assuming an optimal solution exists, any pair of nodes $(v, z)$ with $(v, z) \in V_{2} \times V_{3}$ that are together in a duty in an optimal solution, are connected in $G^{\prime}$. This is true, since these $(v, z)$ apparently satisfy $(v, z) \in E_{2}$, and $w(v)+w(z) \leq O P T$. Thus, a perfect matching exists in $G^{\prime}$, and since we solve an assignment problem in the first stage of $\mathrm{AB}$, we find a perfect matching in the first stage. Then it follows easily that, since $E_{1}$ is complete by assumption, a feasible solution is found by $\mathrm{AB}$.

We now prove the approximation guarantee. Again due to the existence of an optimal solution there exists a perfect matching in $G^{\prime}$ such that there are $k_{1}$ 
edges whose weight in the original graph is bounded by $O P T / 2$. Therefore, a maximum weight matching in $G^{\prime}$ will have weight $k_{1}$. Clearly in the second stage the $k_{1}$ heavy elements from $V_{1}$ can be bottleneck matched with these pairs. The total weight of each of these triples will be bounded by $\frac{3}{2} O P T$. Any other triple will consist of three nonheavy nodes and hence its weight is also bounded by $\frac{3}{2} O P T$. This proves the approximation factor. Finally, we depict in Figure 2 an instance for which this bound is achieved for each $\epsilon>0$, AB can find a solution of value 3 , while $O P T=2+\epsilon$.

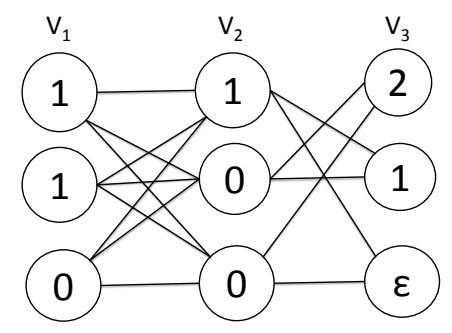

Fig. 2. A worst case instance for heuristic $A B$.

One inconvenience in the preceding discussion is that we assumed knowing the value of OPT. Since we do not know $O P T$, we remedy this by guessing the value of $O P T$, and run $A B$ for each guess. We aim to find the smallest guess for $O P T$ for which $A B$ returns a feasible solution. This smallest guess can be found by a binary search in $\log W$ iterations, where $W$ is the largest weight occurring in the input. The value of the solution returned by $A B$ for this smallest guess of $O P T$, is a $\frac{3}{2}$ approximation. Notice that the overall procedure remains polynomial.

\section{A polynomial time approximation scheme for complete-MBA}

In this section we describe a polynomial time approximation scheme for completeMBA.

First, we choose some $\epsilon$ with $0<\epsilon \leq \frac{1}{2}$ and let $W$ be the largest weight in the instance. Second, we round up each weight $w(v), v \in V$ in the instance to the smallest possible multiple of $\epsilon \cdot W$. Observe that for these rounded-up weights there are $\left\lceil\frac{1}{\epsilon}\right\rceil+1$ distinct values: $0, \epsilon \cdot W, 2 \epsilon \cdot W, \ldots,\left\lceil\frac{1}{\epsilon}\right\rceil \epsilon \cdot W$; we define

$$
K=\left\{0, \epsilon \cdot W, 2 \epsilon \cdot W, \ldots,\left\lceil\frac{1}{\epsilon}\right\rceil \epsilon \cdot W\right\} .
$$


Then, the cartesian product of $m$ sets $K$, referred to as $K^{m}$, is the set of all $m$ tuples $a=(a(1), a(2), \ldots, a(m))$, such that $a(j) \in K$ for each $j=1,2, \ldots, m$. We define, for each $j=1,2, \ldots, m$, and for each $k \in K, A_{j k}=\left\{a \in K^{m}: a(j)=k\right\}$. Further, for each $j=1,2, \ldots, m$, and for each $k \in K$, we define:

Definition 1. $p_{j k}$ equals the number of weights $w\left(v_{i}\right)$ with $v_{i} \in V_{j}$ that - when rounded up - equal $k \epsilon \cdot W$.

Consider now the following integer program that uses variables $x_{a}$ that reflect the number of $m$-tuples consisting of an element from $V_{j}$ with rounded-up weight $a(j)$ for $j=1,2, \ldots, m$. Further, let $c_{a}$ denote the weight of such an $m$-tuple: $c_{a}=a(1)+a(2)+\ldots+a(m)$ for each $a \in K^{m}$.

$$
\begin{array}{cc}
\min & w \\
\text { s.t. } & w \geq c_{a} x_{a} \quad \forall a \in K^{m}, \\
& \sum_{a \in A_{j k}} x_{a}=p_{j k} \forall j=1,2, \ldots, m, k \in K, \\
& x_{a} \text { integral } \quad \forall a \in K^{m} .
\end{array}
$$

Observe that a solution to this integer program corresponds to a solution of complete-MBA. Indeed, a solution to the rounded-up instance of complete-MBA features $n m$-tuples such that each rounded-up weight $k \epsilon \cdot W$ of set $V_{j}$ occurs $p_{j k}$ times. Moreover, both the number of variables and the number of constraints are of $O\left(|K|^{m}\right.$ ), which is polynomial in the input size (for a fixed $\epsilon$ ). Thus we can use Lenstra's algorithm to solve this IP in polynomial time. Observe that this IP is, in fact, nothing else than an (integral) bottleneck axial $m$-index transportation problem (see e.g. Queyranne and Spieksma [12]); it is conceivable that better algorithms than Lenstra's suffice to solve this problem exactly.

The error incurred by a solution to this IP is bounded by the error that is incurred for a single $m$-tuple (or duty), which in turn can be no more than the error induced by rounding the $m$ elements, which equals $m \epsilon W$. In other words

$$
S O L \leq O P T+m \epsilon W \leq(1+m \epsilon) O P T,
$$

where SOL refers to value of the solution found by solving the integer program (1)-(4).

Notice that, given Fact 1, a PTAS for complete-MBA is the strongest result possible (unless $\mathrm{P}=\mathrm{NP}$ ).

\section{Online algorithms for MBA}

In this section we consider the online version of MBA. In the online case, the sets $V_{1}, \ldots, V_{m}$ are revealed one by one in that order. Edges between $V_{i}$ and $V_{i+1}$ are disclosed when $V_{i+1}$ arrives; once $V_{i+1}$ arrives, nodes of $V_{i+1}$ must be immediately and irrevocably matched with the $n$ current $i$-tuples from $V_{1} \times \ldots \times V_{i}$. We call the online version of MBA: online-MBA. 
Online-MBA can be seen as generalisation of online parallel machine scheduling (where jobs with integer weights arrive one by one, and a job is immediately assigned to a machine upon its arrival; the objective is to minimize the makespan). Given an instance of online parallel machine scheduling with $n$ machines, we can construct an instance of online-MBA as follows: For each job $i$, create a set $V_{i}$ with $n-1$ dummy nodes with weight 0 , and a node with weight equal to the weight of job $i$. Each dummy node in $V_{i+1}$ has edges to every node of $V_{i}$, and the node with non-zero weight in $V_{i+1}$ has edges to those nodes in $V_{i}$ which correspond to machines to which job $i+1$ can be assigned.

The Sequential Bottleneck Heuristic (SB), described in Section 3.1 is a natural algorithm for the online-MBA. In fact, it generalizes List Scheduling. However, in the following we show that SB can be arbitrarily bad.

Theorem 5. Sequential Bottleneck Heuristic can be arbitrarily bad for online$M B A$.

Proof. Let $k$ be an odd integer. We construct an instance where SB finds a solution with value $k$, whereas the optimal value equals 1 . We set $m:=2 k-1$ and $n:=k$. Further, $V_{i}:\{\alpha, 0, \ldots, 0\}$ where $\alpha=1$ if $i$ is odd, and 0 otherwise, for $i=1, \ldots, m$. The edge set between $V_{i}$ and $V_{i+1}$ is complete when $i$ is odd, and when $i$ is even, the only edges are: $\left(u_{j}, v_{j}\right) \in V_{i} \times V_{i+1}$, for $j=1, \ldots, n$. Observe that $\mathrm{SB}$ gives a solution with value $k$, whereas the optimum solution has value 1 .

Acknowledgements: We thank Gautam Appa for stimulating discussions, and Gerhard Woeginger for pointing out references.

\section{References}

1. Aringhieri, R., Cordone, R.: The multicommodity multilevel bottleneck assignment problem (2004)

2. Bianco, L., Bielli, M., Mingozzi, A., Ricciardelli, S., Spadoni, M.: A heuristic procedure for the crew rostering problem. European Journal of Operational Research 58, 272-283 (1992)

3. Bodlaender, H., Jansen, K., Woeginger, G.: Scheduling with incompatible jobs. Discrete Applied Mathematics 55, 219-232 (1994)

4. Burkard, R., Dell'Amico, M., Martello, S.: Assignment Problems. SIAM (2009)

5. Cappanera, P., Gallo, G.: A multicommodity flow approach to the crew rostering problem. Operations Research 52, 583-596 (2004)

6. Carraresi, P., Gallo, G.: A multi-level bottleneck assignment approach to the bus drivers rostering problem. European Journal of Operations Research 16, 163-173 (1984)

7. Goossens, D., Polyakovskiy, S., Spieksma, F., Woeginger, G.: The approximability of three-dimensional assignment problems with bottleneck objective. Optimization Letters 4, 4-17 (2010)

8. Hsu, W.L.: Approximation algorithms for the assembly line balancing crew scheduling problem. Mathematics of Operations Research 9, 376-383 (1984) 
9. Jansen, K.: An approximation scheme for bin packing with conflicts. Journal of Combinatorial Optimization 3, 363-377 (1998)

10. Jr., E.C., Yannakakis, M.: Permuting elements within columns of a matrix in order to minimize maximum row sum. Mathematics of Operations Research 9, 384-390 (1984)

11. Klinz, B., Woeginger, G.: A new efficiently solvable case of the three-dimensional axial bottleneck assignment problem. Lecture Notes in Computer Science 1120, 150-162 (1996)

12. Queyranne, M., Spieksma, F.: Approximation algorithms for multi-index transportation problems with decomposable costs. Discrete Applied Mathematics 76, 239-254 (1997) 